\title{
Meeting report \\ Report on the 4th European Breast Cancer Conference, Hamburg, Germany, 16-20 March 2004
}

\author{
Elżbieta Senkus-Konefka and Jacek Jassem
}

\author{
Department of Oncology and Radiotherapy, Medical University of Gdańsk, Poland \\ Corresponding author: Elżbieta Senkus-Konefka, elsenkus@amg.gda.pl \\ Published: 18 May 2004 \\ Breast Cancer Res 2004, 6:148-152 (DOI 10.1186/bcr805) \\ (c) 2004 BioMed Central Ltd
}

\begin{abstract}
The 4th European Breast Cancer Conference, organized under the auspices of the European Organization for Research and Treatment of Cancer Breast Cancer Group, of the European Breast Cancer Coalition (Europa Donna) and of the European Society of Mastology (EUSOMA), was held in Hamburg, Germany on 16-20 March 2004. The leading theme of the conference was partnership among scientists, clinicians, carers, advocates and patients. The present article provides a brief description of the most important conference presentations on molecular biology, epidemiology, prevention, pathology, diagnosis and treatment at all stages of breast cancer.
\end{abstract}

Keywords: biology, breast cancer, diagnosis, patients' advocacy, treatment

The 4th European Breast Cancer Conference was held in Hamburg, Germany on 16-20 March 2004. This joint meeting, organized under the auspices of the European Organization for Research and Treatment of Cancer (EORTC) Breast Cancer Group, of the European Breast Cancer Coalition (Europa Donna) and of the European Society of Mastology (EUSOMA), was attended by 3599 participants (clinicians, scientists and patient advocates) from 82 countries. There were well over 500 presentations on new developments in molecular biology, epidemiology, prevention, diagnosis, treatment, supportive care and the psychosocial and quality-of-life aspects of breast cancer. In addition, there was a special focus on the role of patient advocates and breast cancer support groups. The leading theme of this conference was partnership: the partnership among scientists, clinicians, carers, advocates and patients.

The scientific program was initiated by Martine Piccart from Brussels, with her Emmanuel van der Schueren opening ceremony lecture on tailored adjuvant treatments of early breast cancer. Switching from empirical treatment to individually designed approaches based on specific molecular profiles may soon become a realistic prospect. The gene profiling technology based on microarray assays will hopefully allow for a better selection of candidates for systemic adjuvant treatment and for individualized chemotherapy.

These possibilities are currently being tested in a number of prospective randomized trials, including the EORTC 10994 study (the role of p53 gene mutation in predicting response to anthracycline-based chemotherapy versus taxane-based chemotherapy) and the Trial of Principle (TOP; addressing the impact of topoisomerase II alpha expression on response to anthracycline chemotherapy) both in the setting of locally advanced disease.

The most important question, however, is the putative possibility of avoiding any adjuvant chemotherapy in the subgroups of patients with a 'favorable' gene signature. Such an approach might spare the toxicity and cost of adjuvant chemotherapy in at least $20 \%$ of patients, without influencing the overall prognosis. This hypothesis

ATAC $=$ Arimidex, Tamoxifen Alone or in Combination; EORTC $=$ European Organization for Research and Treatment of Cancer; EUSOMA $=$ European Society of Mastology; IMPACT = IMmediate Preoperative Arimidex, tamoxifen or Combined with Tamoxifen; MINDACT = Microarray for Node Negative Disease may Avoid Chemotherapy; PROACT = PReOperative Arimidex (anastrozole) Compared with Tamoxifen; TOP $=$ Trial of 
will soon be tested in a large prospective randomized trial (Microarray for Node Negative Disease may Avoid Chemotherapy; MINDACT) run by the Breast International Group. This study will compare the outcome of adjuvant chemotherapy in patients assigned to treatment on the grounds of current standard clinical criteria versus assigned according to molecular signature. Microarray technology is also expected to allow for selection of patients for dose-dense chemotherapy or other forms of intensified treatment.

The role of gene expression profiling techniques in recognition of specific cell properties was also addressed in other papers presented at the conference. Biostatistical analysis of gene expression allows for identification of specific 'expression signatures' associated with particular clinical endpoints $[1,2]$. After validation in sufficiently large series of patients, this technology, as well as other emerging genomics and proteomics techniques, may find wide application in the diagnosis of breast cancer. The first example of potentially routine use of gene expression profiling, using a standardized device developed for use at local pathology laboratories (Breast Cancer ProfileChip, Ipsogen SAS, Marseille, France), was presented by French researchers. The use of gene profiling and microarray technology also allows for identification of new subsets of breast cancer with distinct biology and prognosis. A newly recognized important entity, apart from previously described luminal and basal type carcinomas [3], is apocrine carcinoma, which is characterized by positive androgen receptor status and negative estrogen receptor status. These results create the possibility of using androgen suppression in the treatment of selected tumors.

One of the conference highlights was a joint presentation of three recently published randomized trials addressing the role of aromatase inhibitors in the adjuvant treatment of early breast cancer: the Arimidex, Tamoxifen Alone or in Combination (ATAC) study (anastrozole versus tamoxifen versus combination administered for 5 years after local treatment \pm chemotherapy), the MA17 study (5-year administration of letrozole versus placebo after 5 years of tamoxifen treatment), and the most recently published International Exemestane Study (exemestane versus tamoxifen for 2-3 years after 2-3 years of tamoxifen) [4-6]. The latter two studies were presented for the first time at a large scientific meeting. All these studies demonstrated the notable advantage of aromatase inhibitors in terms of disease-free survival. This led to premature termination of these trials and early disclosure of their results.

These recently published studies were also subjected to lively discussion in two Oxford-style debates on the role of aromatase inhibitors in adjuvant treatment of breast cancer and problems related to early presentation of clinical trials. The notion of the outdated role of tamoxifen was proposed and defended by W Jonat from Kiel, Germany (seconded by P Lønning, Bergen, Norway), and was opposed by $\mathrm{K}$ Pritchard from Toronto, Canada (seconded by $\mathrm{H}$ Thornton, Colchester, UK). Although adjuvant aromatase inhibitor administration seems to provide notable benefit, follow-up of these trials was too short to assess reliably the effect of the whole planned intervention.

The only trial that will hopefully provide this information in the future is the International Exemestane Study as, by the time of its closure, more than $90 \%$ of patients had finished protocol treatment. The other two studies were closed prematurely with many patients crossing over to aromatase inhibitor treatment, thus clouding its impact on long-term results (including survival). Furthermore, the data on long-term toxicity of aromatase inhibitors are still missing. Finally, the optimal scheduling of these compounds remains undetermined, as all three studies used them in a different time frame and setting. Some data may suggest that the size of the benefit rises with longer use of tamoxifen preceding the aromatase inhibitor. This can possibly be explained by tamoxifen-induced sensitization of tumor cells to aromatase inhibitors, or by the gradually decreasing effect of tamoxifen (possibly related to its agonistic effect) and the reversal of this process by aromatase inhibitors.

Another controversial and hotly debated issue was early presentation of clinical trials. The opponents included two distinguished biostatisticians (D Berry, Houston, TX, USA, in favor of early disclosure; and R Gelber, Boston, MA, USA, against early disclosure), seconded by two trialists (respectively, J O'Shaughnessy, Dallas, TX, USA and I Tannock, Toronto, Canada). Potential disadvantages of early publications, apart from the previously mentioned incomplete data on late outcomes and toxicity, include their detrimental effect on the conduct of other clinical studies addressing similar issues. Caution must also be paid to the interpretation of early positive results, as this effect may disappear or even reverse with time. Another potential danger is a possible publication bias, as positive studies are more likely to be published sooner. The appearance of a significant difference or an otherwise clinically relevant result early in the course of a trial also creates an important ethical dilemma of releasing these results to patients. Their early switching to potentially beneficial treatment may cloud long-term comparative analysis, and must be weighed against a policy of continuing the study for the benefit of future patients and society.

A series of other important clinical studies on endocrine therapy was presented at the conference. The EORTC 10863 study demonstrated no benefit (in terms of overall survival) of intermittent tamoxifen administration or alter- 
nating tamoxifen/medroxyprogesterone acetate administration, compared with standard continuous tamoxifen administration. The EORTC 10951 trial comparing exemestane and tamoxifen in the first-line treatment of advanced disease demonstrated an apparent superiority of exemestane in terms of response rate (44\% versus $29 \%$ ) and time to progression (median, 10.9 months and 6.7 months, respectively). At the same time, no adverse effect on lipid profiles, observed in studies of nonsteroidal aromatase inhibitors, was found. Results of the EORTC 10901 study including more than 1800 patients confirmed prospectively the disease-free survival advantage associated with a 3-year tamoxifen treatment following adjuvant chemotherapy in operable breast cancer. The IMPACT (IMmediate Preoperative Arimidex, tamoxifen or Combined with Tamoxifen) and PROACT (PReOperative Arimidex (anastrozole) Compared with Tamoxifen) trials failed to demonstrate a significant advantage of preoperative anastrozole compared with tamoxifen in locally advanced/ large operable tumors, although some benefit may be confined to selected endpoints and subgroups of patients.

The area of clinical research arousing major interest over the past years and widely discussed during the conference was primary chemotherapy. With efficacy proven to be equivalent to adjuvant therapy $[7,8]$, this setting not only offers increased rates of breast conservation, but also creates an ideal experimental model for in vivo predictive assays of various treatments including targeting factors. The most important areas of clinical research include the use of noncross-resistant taxanebased regimens of chemotherapy $[9,10]$, dose-dense chemotherapy schedules [11] and the use of aromatase inhibitors. The question remains, however, whether particular surrogate endpoints (such as the rate of pathological complete remission) are reliable predictors of late results. Such correlations might facilitate and significantly shorten the evaluation of adjuvant systemic therapies. New research directions in this group of patients include development of diagnostic tools for early prediction of response (such as magnetic resonance imaging or positron emission tomography) and monitoring of treatment-induced molecular changes in tumor cells.

An important part of the discussion on new chemotherapy strategies included the dose-dense approach, a strategy believed to offer the chance of overcoming tumor regrowth between consecutive cycles. Indeed, early results of the Intergroup Trial C9741/Cancer and Leukemia Group B Trial 9741 demonstrated a significant improvement in disease-free and overall survival in node-positive patients administered dose-dense adjuvant chemotherapy [11]. Other approaches to overcoming tumor resistance include the incorporation of noncross-resistant drugs (in particular, taxanes). Of interest were the updated results disease-free and overall survival advantage of the taxanebased regimen (docetaxel + doxorubicin + cyclophosphamide) over the standard 5-fluorouracil + doxorubicin + cyclophosphamide regimen in adjuvant treatment of nodepositive patients.

A landmark paper by Slamon and colleagues [12] was the first to demonstrate a significant advantage associated with the addition of trastuzumab to paclitaxel in advanced breast cancer. New evidence for the role of trastuzumab comes from the updated results of the M77001 study, showing 8-month median survival improvement in Her2positive metastatic disease patients administered trastuzumab and docetaxel, compared with docetaxel alone. The results of ongoing studies addressing the use of trastuzumab in the adjuvant setting are awaited with great interest.

Changing trends in breast cancer treatment include better understanding of the different biology of two main breast cancer subtypes: hormone-responsive breast cancer and hormone-independent breast cancer. This phenomenon is reflected by higher rates of response to chemotherapy in hormone-independent tumors [9] and, on the contrary, in a shift away from adjuvant chemotherapy in receptorpositive patients. Data were presented on the relationship between hormone receptor level and benefit from addition of chemotherapy to tamoxifen in the adjuvant treatment, demonstrating no advantage from combined treatment in patients with moderate and high hormone receptor concentrations.

A few studies recently indicated higher response rates and/or prolonged disease-free survival associated with aromatase inhibitor therapy compared with tamoxifen in progesterone receptor-negative tumors and ErbB-1positive and/or ErbB-2 positive tumors [13]. These findings may provide a basis for the selection of relatively small subgroups of patients deriving a large benefit from adjuvant aromatase inhibitors, saving society the high cost of treatment of the remaining patients, in whom tamoxifen may prove equally effective.

An intriguing, although not fully understood, piece of information came from the Dutch study on cardiovascular mortality following breast cancer treatment. Although in the whole population of breast cancer survivors the risk of cardiovascular death was within the range of normal expectancy, cardiovascular mortality was significantly decreased in a subgroup of nonirradiated patients in comparison with the general population. A possible explanation for this phenomenon is that the risk profile for breast cancer or a healthier lifestyle after breast cancer diagnosis may be protective against cardiovascular morbidity.

Two interesting and important observations regarding standards of breast cancer care were presented. The first, 
coming from a large Canadian population-based study, confirmed decreased mortality (hazard ratio, 0.7) in patients managed in concordance with accepted guidelines and treated at centers dealing with large numbers of cases. Particular gain (hazard ratio, 0.4) was attributed to participation in clinical trials. The other analysis, including postmenopausal patients treated within the International Exemestane Study, demonstrated large differences in the frequency of breast conservation rates between countries, ranging from $2 \%$ in Poland to $72 \%$ in France. Possible explanations for this observation include differences in stage distribution related to efficacy of nationwide screening programs, tradition, and surgeons' and patients' attitudes. Further analyses are warranted to fully elucidate this phenomenon.

The conference was, as usual, concluded by the interactive plenary session on critical issues in breast cancer, leading to the development of the Hamburg Statement. This document will set out the directions for breast cancer research and care for the next 2 years. The first problem discussed was threats and constraints to academic research, possibly leading to a shift towards industryoriented studies and to abandoning other areas of clinical research, such as diagnosis, surgery and radiotherapy. A call was made for more determined financial and structural support for academic research, facilitation of free circulation of tissue and blood samples within the European Union for research purposes, and a greater involvement of patients and consumers in research planning and monitoring [14].

Another issue addressed in this session was the limited consideration of special needs of elderly patients, reflected by their restricted inclusion in clinical trials. It was postulated that participation in these trials should be based on physiological status rather than on age, and that no upper age limit should be laid down in the design of standard prevention and treatment plans. Recommendations were also made on the need of professional care for high-risk women and on providing full free-of-charge genetic counseling and testing for women with a strong family history of breast cancer [14].

Finally, a plea for improvements in care for breast cancer survivors was made. Apart from detecting local tumor recurrence and second malignancies, this should include psychological support and the management of late treatment sequelae [14].

The 5th European Breast Cancer Conference will be held in Nice, France, on 21-25 March 2006.

\section{Competing interests}

None declared.

\section{References}

1. van de Vijver MJ, He YD, van't Veer LJ, Dai H, Hart AA, Voskuil DW, Schreiber GJ, Peterse JL, Roberts C, Marton MJ, Parrish M, Atsma D, Witteveen A, Glas A, Delahaye L, van der Velde T, Bartelink H, Rodenhuis S, Rutgers ET, Friend SH, Bernards R: A gene-expression signature as a predictor of survival in breast cancer. N Engl J Med 2002, 347:1999-2009.

2. Pusztai L, Ayers M, Simmans FW, Damokosh A, Hess K, Valero V, Clark E, Ross J, Hortobagyi GN, Stec J: Emerging science: prospective validation of gene expression profiling-based prediction of complete pathologic response to neoadjuvant paclitaxel/FAC chemotherapy in breast cancer. Proc $\mathrm{Am}$ Soc Clin Oncol 2003, 22:1.

3. Sorlie T, Perou CM, Tibshirani R, Aas T, Geisler S, Johnsen H, Hastie T, Eisen MB, van de Rijn M, Jeffrey SS, Thorsen T, Quist H, Matese JC, Brown PO, Botstein D, Eystein Lonning P, BorresenDale AL: Gene expression patterns of breast carcinomas distinguish tumor subclasses with clinical implications. Proc Natl Acad Sci USA 2001, 98:10869-10874.

4. Baum M, Buzdar A, Cuzick J, Forbes J, Houghton J, Howell A, Sahmoud T, The ATAC (Arimidex, Tamoxifen Alone or in Combination) Trialists' Group: Anastrozole alone or in combination with tamoxifen versus tamoxifen alone for adjuvant treatment of postmenopausal women with early-stage breast cancer: results of the ATAC (Arimidex, Tamoxifen Alone or in Combination) trial efficacy and safety update analyses. Cancer 2003, 98:1802-1810.

5. Goss PE, Ingle JN, Martino S, Robert NJ, Muss HB, Piccart MJ, Castiglione M, Tu D, Shepherd LE, Pritchard KI, Livingston RB, Davidson NE, Norton L, Perez EA, Abrams JS, Therasse P, Palmer MJ, Pater JL: A randomized trial of letrozole in postmenopausal women after five years of tamoxifen therapy for early-stage breast cancer. N Engl J Med 2003, 349:1793-1802.

6. Coombes RC, Hall E, Gibson LJ, Paridaens R, Jassem J, Delozier T, Jones SE, Alvarez I, Bertelli G, Ortmann O, Coates AS, Bajetta E, Dodwell D, Coleman RE, Fallowfield LJ, Mickiewicz E, Andersen J, Lonning PE, Cocconi G, Stewart A, Stuart N, Snowdon CF, Carpentieri M, Massimini G, Bliss JM; Intergroup Exemestane Study: A randomized trial of exemestane after two to three years of tamoxifen therapy in postmenopausal women with primary breast cancer. N Engl J Med 2004, 350:1081-1092.

7. Fisher B, Brown A, Mamounas E, Wieand S, Robidoux A, Margolese RG, Cruz AB Jr, Fisher ER, Wickerham DL, Wolmark N, DeCillis A, Hoehn JL, Lees AW, Dimitrov NV: Effect of preoperative chemotherapy on local-regional disease in women with operable breast cancer: findings from National Surgical Adjuvant Breast and Bowel Project B-18. J Clin Oncol 1997, 15:2483-2493.

8. van der Hage JA, van de Velde CJ, Julien JP, Tubiana-Hulin M, Vandervelden C, Duchateau L: Preoperative chemotherapy in primary operable breast cancer: results from the European Organization for Research and Treatment of Cancer trial 10902. J Clin Oncol 2001, 19:4224-4237.

9. Bear HD, Anderson S, Brown A, Smith R, Mamounas EP, Fisher B, Margolese R, Theoret H, Soran A, Wickerham DL, Wolmark N: National Surgical Adjuvant Breast and Bowel Project Protocol B27: The effect on tumor response of adding sequential preoperative docetaxel to preoperative doxorubicin and cyclophosphamide: preliminary results from National Surgical Adjuvant Breast and Bowel Project Protocol B-27. J Clin Oncol 2003, 21:4165-4174.

10. Smith IC, Heys SD, Hutcheon AW, Miller ID, Payne S, Gilbert FJ, Ah-See AK, Eremin O, Walker LG, Sarkar TK, Eggleton SP, Ogston KN: Neoadjuvant chemotherapy in breast cancer: significantly enhanced response with docetaxel. J Clin Oncol 2002, 20:1456-1466.

11. Citron ML, Berry DA, Cirrincione C, Hudis C, Winer EP, Gradishar WJ, Davidson NE, Martino S, Livingston R, Ingle JN, Perez EA, Carpenter J, Hurd D, Holland JF, Smith BL, Sartor Cl, Leung EH, Abrams J, Schilsky RL, Muss HB, Norton L: Randomized trial of dose-dense versus conventionally scheduled and sequential versus concurrent combination chemotherapy as postoperative adjuvant treatment of node-positive primary breast cancer: first report of Intergroup Trial C9741/Cancer and Leukemia Group B Trial 9741. J Clin Oncol 2003, 21:1431-1439.

12. Slamon DJ, Leyland-Jones B, Shak S, Fuchs H, Paton V, Bajamonde A, Fleming T, Eiermann W, Wolter J, Pegram M, Baselga J, 
Norton L: Use of chemotherapy plus a monoclonal antibody against HER2 for metastatic breast cancer that overexpresses HER2. N Engl J Med 2001, 344:783-792.

13. Ellis MJ, Coop A, Singh B, Mauriac L, Llombert-Cussac A, Janicke F, Miller WR, Evans DB, Dugan M, Brady C, Quebe-Fehling E, Borgs $M$ : Letrozole is more effective neoadjuvant endocrine therapy than tamoxifen for ErbB-1- and/or ErbB-2-positive, estrogen receptor-positive primary breast cancer: evidence from a phase III randomized trial. $J$ Clin Oncol 2001, 19:38083816.

14. Jassem J, Buchanan M, Jänicke F, Baum M, Cataliotti L, Piccart M, Rutgers EJT, Costa A: The Hamburg Statement: the partnership driving the European agenda on breast cancer. Eur $J$ Cancer (in press) [http://www.fecs.be/conferences/ebcc4/ pressreleases/Hamburgstatement.doc]. 